\title{
Confirmed Case of Buruli Ulcer, Senegal, 2018
}

\section{Grace Anne Turner, Abdoulave Seck, Assane Dieng, Saër Diadie, Babacar Ndiaye, Tabitha D. van Imeerzeel, Moussa Diallo, Marie Kempf, Raymond Bercion, Cheikh Saad-Bouh Boye}

Author affiliations: Keru Yakaar, Dakar, Senegal (G.A. Turner, T.D. van Imeerzeel); University of Cheikh Anta Diop, Dakar (A. Seck, A. Dieng, S. Diadie, M. Diallo, C.S.-B. Boye); Pasteur Institute, Dakar (A. Seck, B. Ndiaye, R. Bercion); University of Angers, Angers, France (M. Kempf); University Hospital Center, Angers (M. Kempf); University Hospital Center Dantec, Dakar (C.S.-B. Boye)

DOI: https://doi.org/10.3201/eid2503.180707

Buruli ulcer is a necrotizing skin disease caused by Mycobacterium ulcerans and is usually associated with tropical climates and exposure to slow-moving or stagnant water. We report a case of Buruli ulcer that may have originated in an urban semiarid area of Senegal.

$\mathrm{I}$ n January 2018, a 14-year-old boy came to an urban clinic in Dakar, the capital of Senegal, with a 2-week history of skin lesions. He had a $1 \times 1 \mathrm{~cm}$ ulcerous erosion over a $6 \times 16 \mathrm{~cm}$ painful edematous lesion on his right calf; he was febrile, with a temperature of $38.5^{\circ} \mathrm{C}$. He was initially treated for cellulitis with amoxicillin and clavulanate acid, along with wound care. Two days later, the lesion had evolved. Debridement revealed considerable necrotic subcutaneous tissue extending $1-3 \mathrm{~cm}$ under the epidermal edge. The most proximal of the 3 ulcers had a diameter of $1 \mathrm{~cm}$, the next measured $5 \times 6 \mathrm{~cm}$, and the last was an Lshaped lesion measuring $6 \times 28 \mathrm{~cm}$, running from midcalf to toes. Infection with Mycobacterium ulcerans was suspected because of rapid tissue necrosis, classic undermining edges, patient age, location of the lesions, and failure of standard care (Appendix Figure 1, http://wwwnc.cdc.gov/ EID/article/25/3/18-0707-App1.pdf).

The patient was admitted to the hospital and treated with parenteral gentamicin, oral metronidazole, and wound care. The wound bed was swabbed; culture revealed Acinetobacter and Pseudomonas. Antimicrobial drug therapy was changed to parenteral gentamicin and oral ciprofloxacin. Four swab specimens were obtained from the wound, and quantitative real-time PCR assay targeting the IS2404 putative transposase gene and the mycolactone polyketide synthase gene confirmed the presence of $M$. ulcerans. Targeting IS2404 is considered the diagnostic standard for Buruli ulcer (1). Targeting IS2404 PCR analysis for
M. tuberculosis and negative controls were both negative (Appendix). A skin graft was performed, and the patient was discharged and given rifampin/isoniazid, ciprofloxacin, and wound care.

The patient had been born in rural Guinea-Conakry and moved to Senegal 3 years before his illness. His mother reported that he had been fully vaccinated, although no records remain. He moved to Senegal in 2015 and lived in Dakar for 18 months, then moved east to the semiarid area of Diourbel to attend Koranic school for another 18 months. He denied engaging in any agricultural or mining activities or bathing, washing, or swimming in bodies of fresh water during his 3 years in Senegal. He also denied returning to Guinea-Conakry or other travel since his arrival in Senegal. In GuineaConakry, he had been involved in agricultural activities, including rice farming. The family does not use mosquito nets, and he reported occasional insect bites.

Worldwide, Buruli ulcer is the third most common mycobacterial infection, inflicting debilitating cost and social stigma on patients and their families $(2,3)$. The highest incidence of Buruli ulcer is found in tropical or subtropical sub-Saharan Africa, but 2 cases have been reported in Mali, a semiarid country not usually associated with Buruli ulcer (3-5). The only other known case of Buruli ulcer in Senegal was in a traveler from Europe who had been building canoes in fresh water along the tropical Senegal-Guinea border (6).

The mode of transmission of M. ulcerans is poorly understood and may vary by region. The bacterium has been found in aquatic environments, animals, and insects. Animal reservoirs and insect vectors have been proposed, but no definitive vector has been identified (7). A systematic review found that poor wound care, living or working near aquatic environments, and failure to wear protective clothing (long pants and long-sleeved shirts) were risk factors associated with $M$. ulcerans infection. Results among other researchers searching for risk factors have been contradictory (8). The reported incubation period ranges between 34 and 264 days, with a mean of 4.5 months (9). A multicenter study in West Africa demonstrated no significant evidence of protection from $M$. ulcerans infection after bacillus Calmette-Guérrin vaccination (10).

This case of Buruli ulcer is noteworthy because it is a confirmed case originating in a semiarid region of West Africa, suggesting that the endemic area of this disease is poorly defined or changing. The patient appears to have contracted the disease in Senegal without the usual waterrelated risk factors, although he was exposed to insect bites. It is possible but unlikely that he contracted the disease in Guinea-Conakry 3 years earlier, which would mean that he had an incubation period 2 years longer than any previously reported cases. There is no evidence to suggest his possible bacillus Calmette-Guérrin vaccination delayed wound development. 
This case illustrates the need to better define the geographic extent and modes of transmission of this debilitating disease so that primary control measures can be identified. In addition, health workers must be provided with the training and tools to diagnose and treat M. ulcerans. Research into a point-of-care diagnostic test is needed so that timely treatment can minimize disability and costs to the family.

\section{Acknowledgments}

Thanks to Emily Duecke, Sidy Ba, Carlos Bleck, and Teunella Wolters for their sharp clinical skills and therapeutic efforts on behalf of this patient.

\section{About the Author}

Ms. Turner is a family nurse practitioner living and working in Dakar, Senegal. Her background includes trauma and pediatric primary care in high-income and low-income countries.

\section{References}

1. Sakyi SA, Aboagye SY, Otchere ID, Yeboah-Manu D. Clinical and laboratory diagnosis of Buruli ulcer disease: a systematic review. Canadian J Infect Dis Med Microbiol. 2016;2016:5310718. http://dx.doi.org/10.1155/2016/5310718

2. Guarner J. Buruli ulcer. Review of a neglected skin mycobacterial disease. J Clin Microbiol. 2018;56:01507-17.

3. World Health Organization. Treatment of Mycobacterium ulcerans disease (Buruli ulcer). Guidance for health workers. Geneva: The Organization; 2010. p. 1-3.

4. Ezzedine K, Pistone T, Guir V, Malvy D. Painful Buruli ulcer in a Malian visitor to France. Acta Derm Venereol. 2010;90:424. http://dx.doi.org/10.2340/00015555-0862

5. Bessis D, Kempf M, Marsollier L. Mycobacterium ulcerans disease (Buruli ulcer) in Mali: a new potential African endemic country. Acta Derm Venereol. 2015;95:489-90. http://dx.doi.org/ 10.2340/00015555-1942

6. Ezzedine K, Pistone T, Cottin J, Marsollier L, Guir V, Malvy D. Buruli ulcer in long-term traveler to Senegal. Emerg Infect Dis. 2009;15:118-9. http://dx.doi.org/10.3201/ eid1501.080123

7. Merritt RW, Walker ED, Small PLC, Wallace JR, Johnson PDR, Benbow ME, et al. Ecology and transmission of Buruli ulcer disease: a systematic review. PLoS Negl Trop Dis. 2010;4:e911. http://dx.doi.org/10.1371/journal.pntd.0000911

8. Jacobsen KH, Padgett JJ. Risk factors for Mycobacterium ulcerans infection. Int J Infect Dis. 2010;14:e677-81. http://dx.doi.org/10.1016/j.ijid.2009.11.013

9. Trubiano JA, Lavender CJ, Fyfe JAM, Bittmann S, Johnson PDR. The incubation period of Buruli ulcer (Mycobacterium ulcerans infection). PLoS Neg1 Trop Dis. 2013;7:e2463. http://dx.doi.org/ 10.1371/journal.pntd.0002463

10. Phillips RO, Phanzu DM, Beissner M, Badziklou K, Luzolo EK, Sarfo FS, et al. Effectiveness of routine BCG vaccination on Buruli ulcer disease: a case-control study in the Democratic Republic of Congo, Ghana and Togo. PLoS Negl Trop Dis. 2015;9:e3457. http://dx.doi.org/10.1371/journal.pntd.0003457

Address for correspondence: Grace Anne Turner, 1609 Watkins St, Lake Charles, LA 70601, USA; email: gaturner@gmail.com

\section{Management of Patients with Candida auris Fungemia at Community Hospital, Brooklyn, New York, USA, 2016-2018}

\author{
Jenny YeiSol Park, ${ }^{2}$ Nicole Bradley, ${ }^{3}$ \\ Steven Brooks, Sibte Burney, Chanie Wassner
}

DOI: https://doi.org/10.3201/eid2503.180927

Candida auris is an emerging fungus that can cause invasive infections. It is associated with high mortality rates and resistance to multiple classes of antifungal drugs and is difficult to identify with standard laboratory methods. We describe the management and outcomes of 9 patients with $C$. auris fungemia in Brooklyn, New York, USA.

Csiv andida auris is an emerging fungus that can cause invasive infections associated with high mortality rates and is often resistant to multiple classes of antifungal drugs. Risk factors for infection include nursing home exposure; invasive devices, such as tracheostomy tubes or percutaneous endoscopic gastrostomy tubes; immunocompromised status; and use of broad-spectrum antimicrobial drugs (1). On the basis of limited data available, echinocandins are recommended as initial therapy for $C$. auris infection (2). We review the management of 9 case-patients who had $C$. auris fungemia at a 300-bed community hospital, attached to a 450-bed nursing home, in Brooklyn, NY, USA. There have been 9 occurrences of C. auris fungemia at this institution since 2016.

Our case series demonstrates the complex patient population at risk for invasive infection with $C$. auris. Patients infected were generally $>70$ years of age and had multiple chronic concurrent conditions (Appendix Table, https://wwwnc.cdc.gov/EID/article/25/3/18-0927-App1. pdf). Most patients came from nursing homes, and more than half had invasive devices, such as tracheostomies or

Author affiliation: Kingsbrook Jewish Medical Center, Brooklyn, New York, USA

DOI: https://doi.org/10.3201/eid2503.180927

${ }^{1}$ Preliminary results from this study were presented as a poster presentation at the American Society of Health-System Pharmacists Midyear Meeting, December 3-7, 2017, Orlando, Florida, USA.

${ }^{2}$ Current affiliation: State University of New York Downstate Medical Center, Brooklyn, New York, USA.

${ }^{3}$ Current affiliation: St. John's University College of Pharmacy and Health Sciences, Queens, New York, USA. 